\title{
Optical fiber taper coupling and high-resolution wavelength tuning of microdisk resonators at cryogenic temperatures
}

\author{
Kartik Srinivasan ${ }^{\text {a) }}$ and Oskar Painter \\ Center for the Physics of Information, California Institute of Technology, Pasadena, California 91125 \\ and Department of Applied Physics, California Institute of Technology, Pasadena, California 91125
}

(Received 14 November 2006; accepted 10 December 2006; published online 19 January 2007)

\begin{abstract}
A system for studying microcavity resonators at cryogenic temperatures $(\sim 10 \mathrm{~K})$ through evanescent coupling via optical fiber taper waveguides is reported, and efficient fiber coupling to AlGaAs microdisk cavities with embedded quantum dots is demonstrated. As an immediate application of this tool, the authors study high-resolution tuning of microdisk cavities through nitrogen gas adsorption, as first discussed by Mosor et al. [Appl. Phys. Lett. 87, 141105 (2005)]. By proper regulation of the nitrogen gas flow and delivery of the gas to the sample surface, continuous tuning can be achieved with modest gas flows, and overall wavelength shifts as large as $4 \mathrm{~nm}$ are achieved. () 2007 American Institute of Physics. [DOI: 10.1063/1.2431719]
\end{abstract}

Solid-state systems involving a semiconductor microcavity coupled to a semiconductor quantum $\operatorname{dot}^{1}(\mathrm{QD})$ offer a promising implementation of cavity quantum electrodynamics $^{2}$ (cQED) for quantum information processing and computing applications. In addition to demonstrations of vacuum Rabi splitting in the emission spectrum of a QDmicrocavity system, ${ }^{3-5}$ the quality factor $(Q)$ of wavelengthscale III-V semiconductor microcavities has recently exceeded $10^{5,}, 7$ paving the way for cQED experiments in which the coherent QD-photon coupling rate can greatly exceed the system's dissipative rates. Also important is the development of an efficient interface through which the microscopic cavity field can be accessed from macroscopic optics. Silica optical fiber tapers, initially used as effective inputoutput couplers for silica microcavities, ${ }^{8,9}$ have recently been used to couple to high refractive index microcavities, ${ }^{10}$ including AlGaAs microdisk cavities with embedded QDs. ${ }^{6}$

Here, we report on the development of a system that extends our previous work, done at room temperature and pressure, to the high vacuum $\left(\sim 10^{-6}\right.$ Torr $)$, cryogenic $(\sim 10 \mathrm{~K})$ environments in which QD-based cQED experiments are done. ${ }^{3-5}$ This system is used to interrogate wavelength-scale GaAs/AlGaAs microdisk cavities containing self-assembled InAs QDs, with information such as the cavity transmission and QD emission spectrum obtained. In the second part of this letter, we build on the work of Refs. 11 and 12, using nitrogen $\left(\mathrm{N}_{2}\right)$ gas adsorption to tune the resonant wavelength of microcavities in a cryogenic environment. By proper regulation of the $\mathrm{N}_{2}$ flow and delivery of the gas near the sample surface, potential difficulties discussed in Ref. 11 are overcome, and reproducible, highresolution tuning is achieved. Furthermore, the fiber taper coupling allows for detailed investigation of the $\mathrm{N}_{2}$ adsorption process.

The fiber tapers used are single mode optical fibers that have been heated and stretched so that their central region has a minimum diameter of $\sim 1 \mu \mathrm{m}$. Potential difficulties in extending fiber taper coupling to a high vacuum, cryogenic environment include the mechanical stability of the fiber taper assembly, the lack of viscous air-damping of fiber taper vibrations, and the mechanical and optical stability of the

\footnotetext{
${ }^{a)}$ FAX: (626) 795-7258; electronic mail: kartik@caltech.edu
}

fiber taper itself under repeated temperature cycling. Figure 1 depicts the setup we have developed, where the sample and fiber taper reside inside a modified Janis ST-500 continuous flow, liquid He cryostat. A Teflon-based compression fitting ${ }^{13}$ is used to feed the two optical fiber pigtails of the fiber taper from the interior vacuum to the exterior of the cryostat. The fiber taper is held in a "u-shaped" configuration to provide self-tensioning of the taper. Coarse alignment of the taper to the microcavity is achieved by positioning the microcavity sample using slip-stick $Y-Z$ piezopositioners with a displacement range of several millimeters. Fine adjustment in the taper position is provided by an $X-Y-Z$ piezoelectric flexure stage with a maximum displacement of several microns at $4.2 \mathrm{~K}$. A thermally conductive pathway between the sample and the cold finger is provided by copper braids that connect the top sample mount to the cold finger. This ensures that the sample can get to the requisite low temperature $(<15 \mathrm{~K})$, which is measured by a silicon diode that is affixed to the top sample mount.

The devices we study are small diameter $(D=2-4.5 \mu \mathrm{m}) \mathrm{GaAs} / \mathrm{AlGaAs}$ microdisks that contain a single layer of InAs QDs (room temperature ground state emission at $\sim 1317 \mathrm{~nm}$ ), as discussed elsewhere. ${ }^{6}$ The cryostat is cooled to a sample temperature of $14 \mathrm{~K}$, and during this process, no additional loss in the optical fiber taper transmission is observed (typical total insertion loss is 10\%-50\% depending on the taper tension). The taper is positioned in the near field of the microdisk under study using the pi-

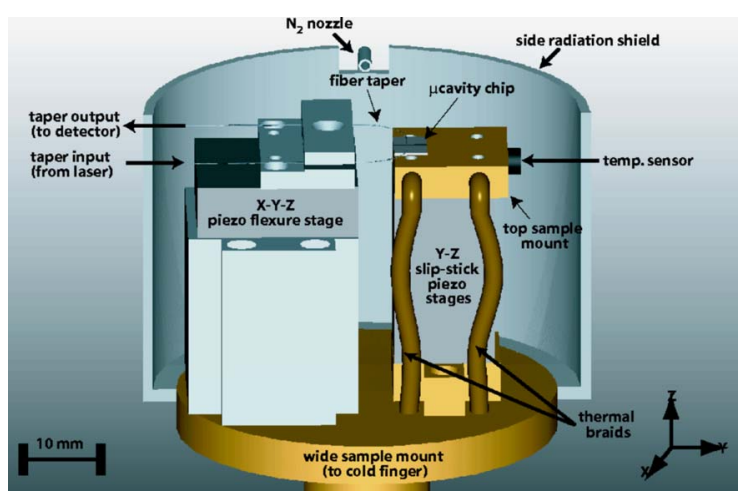

FIG. 1. Cutaway diagram of the cryostat interior, illustrating the arrangement of the fiber taper, sample, and $\mathrm{N}_{2}$ flow nozzle. 

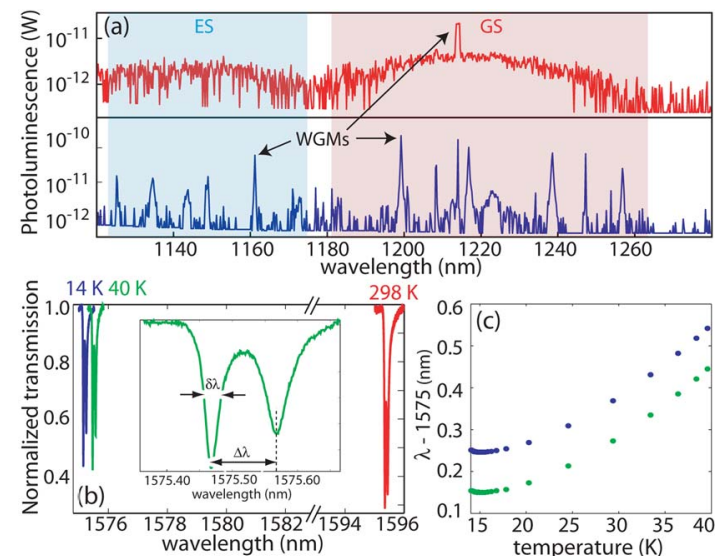

FIG. 2. (a) Comparison of photoluminescence data from an embedded layer of QDs in a $D=4.5 \mu \mathrm{m}$ disk, using free-space collection (top) and fibertaper-based collection (bottom). Resolution bandwidth is 0.1 (1) $\mathrm{nm}$ for the fiber taper (free space) collection spectra. [(b) and (c)] Temperature tuning data for the $\mathrm{TE}_{1,20} \mathrm{WGM}$.

ezostage configuration described above. An adjustable airgap may be maintained between taper and disk or the taper may be placed into direct contact with the disk, depending on the level of cavity loading desired (anywhere from under- to overcoupled is possible). Accuracy in the taper-disk gap is limited only by vibration-induced fluctuations in the taper position (tens of nanometers in our current setup). In general, we have found that the taper can remain in a fixed coupling position (at room or cryogenic temperatures) for times as long as several hours. The fiber taper was also unaffected by repeated temperature cycling. As described in detail in Ref. 14, the taper can be used to enhance the collection efficiency of light emitted from microdisk whispering gallery modes (WGMs) by nearly two orders of magnitude over normalincidence free-space collection. Figure 2 compares fiber taper and free-space collection of the low temperature $(T$ $=14 \mathrm{~K}$ ) emission from a $D=4.5 \mu \mathrm{m}$ microdisk that is optically pumped with $\sim 100 \mu \mathrm{W}$ of incident power from an $830 \mathrm{~nm}$ laser diode. The collected power and number of cavity modes observed in the taper-collected spectrum greatly exceed that obtained by free-space collection, with emission into WGMs from the ground and excited state manifolds of the QDs clearly visible.

More than just an efficient collection optic, the fiber taper may also be used to optically probe and excite the cavity-QD system in a highly efficient manner. Here we use the fiber taper to monitor the transmission properties of the cavity modes of the microdisk as a function of temperature, and as described below, during cavity mode tuning experiments involving $\mathrm{N}_{2}$ gas adsorption. To this end, a scanning tunable laser (linewidth $<5 \mathrm{MHz}$ ) is connected to the fiber taper input and the wavelength-dependent taper output transmission is monitored with a photodetector. The polarization state of the fiber taper mode is achieved through a polarization controller inserted between the laser and taper input. Figure 2(b) shows the transmission spectra of a cavity mode in a $D=4.5 \mu \mathrm{m}$ disk in the $1500 \mathrm{~nm}$ band. ${ }^{15}$ The cavity mode wavelength is seen to shift approximately $20 \mathrm{~nm}$ as the temperature is reduced from 298 to $14 \mathrm{~K}$ as a result of the decrease in the AlGaAs disk refractive index. As noted in our previous work (Ref. 6 and references therein), the high- $Q$ modes often appear as a doublet [inset of Fig. 2(b)] due to surface roughness on the microdisk which couples the iniDownloaded 29 Jan 2007 to 131.215 .225 .173 . Redistribution subject

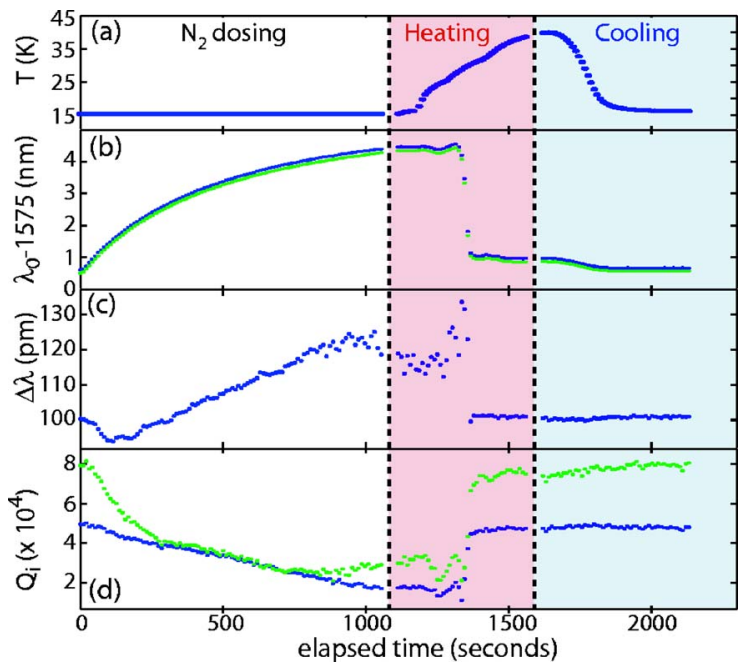

FIG. 3. Tuning data ( $\mathrm{TE}_{1,20}$ mode in a $D=4.5 \mu \mathrm{m}$ disk) during $\mathrm{N}_{2}$ dosing, sample heating, and sample cooldown phases. (a) Sample temperature $(T)$, (b) resonant mode wavelengths $\left(\lambda_{0}\right)$, (c) doublet mode splitting $(\Delta \lambda)$, and (d) intrinsic cavity $Q\left(Q_{i}\right)$. The green (blue) curve is for the short (long) wavelength doublet mode.

tially degenerate WGMs into frequency-split standing wave modes.

As discussed in many other works, the small tuning range $[0.3 \mathrm{~nm}$ for $T=14-40 \mathrm{~K}$ in Fig. 2(c)] afforded by thermal tuning is a significant limitation in cQED experiments due to the imprecise spectral positioning of QD exciton peaks and cavity modes during growth and fabrication. To overcome this difficulty, Mosor et al. utilized noble gas adsorption on the sample surface to achieve postfabrication shifts in a photonic crystal cavity of up to $5 \mathrm{~nm} .{ }^{11}$ Here, we apply this method to tune the resonances of our microdisk cavities while using the fiber taper probe to monitor their behavior. Reference 11 achieves wavelength tuning in discrete steps by filling a secondary chamber with gas (Xe or $\mathrm{N}_{2}$ ) until a desired pressure is reached, releasing that volume into the cryostat, and then repeating. The authors found that the fill pressure must lie within a vary narrow range, below which no tuning occurs and above which excessively fast tuning occurs. To improve upon the tuning resolution and repeatability, we have made two key modifications. Rather than introduce the gas through the cryostat vacuum line, we inject it through a 1/16 in. tube (inner diameter of $0.56 \mathrm{~mm}$ ) that is routed into an opening in the top of the side radiation shield (Fig. 1), so that gas can be locally delivered with line of sight to the sample. Next, instead of introducing the gas into the cryostat through repeated cycles, we fill an external chamber $(V=0.11)$ until a fixed pressure is reached (10 torr) and then release it into the cryostat using a metering valve to control the flow rate. We monitor the cavity mode transmission spectrum and use a shut-off valve to stop the gas flow when a desired wavelength shift is achieved (the shut-off and metering valves are positioned as close as possible to the cryostat to minimize dead volume between themselves and the end of the injection nozzle).

Tuning data obtained using high purity (99.9995\%) $\mathrm{N}_{2}$ gas are shown in Fig. 3 for the microdisk mode studied in Figs. 2(b) and 2(c), with the taper in contact with the disk edge. Transmission spectra of the taper-coupled microdisk were recorded every $10 \mathrm{~s}$ over the entire tuning cycle, which included an initial $\mathrm{N}_{2}$ dosing period, a subsequent $\mathrm{N}_{2}$ desorption phase accomplished by heating the sample with a resisto AIP license or copyright, see http://apl.aip.org/apl/copyright.jsp 


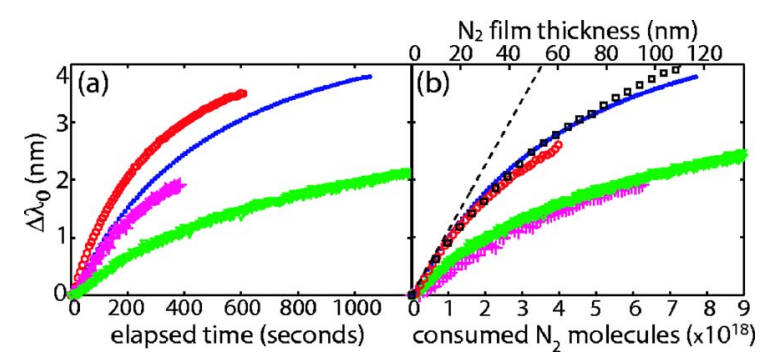

FIG. 4. Wavelength shift $\left(\Delta \lambda_{0}\right)$ vs (a) time and (b) $\mathrm{N}_{2}$ consumption (thickness) for different $\mathrm{N}_{2}$ flow rates. $(\bigcirc)$ and (.) correspond to measurements of the mode in Fig. $3(D=4.5 \mu \mathrm{m})$, while $(+)$ and $(\nabla)$ are for a WGM at $\lambda_{0}$ $=1255 \mathrm{~nm}$ in a $D=2.0 \mu \mathrm{m}$ microdisk. The average flow rates are $(\bigcirc),(\cdot)$, $(+),(\nabla)=(13.0,7.4,16.0,5.5) \times 10^{15} \mathrm{~N}_{2}$ molecules/s. The $\square(-)$ curve in (b) corresponds to FEM (perturbation) simulation of the tuning vs film thickness for the $D=4.5 \mu \mathrm{m} \mathrm{TE}_{1,20}$ WGM.

tive heater, and a final cooldown period. The cavity transmission spectra were fitted using a standard doublet model, ${ }^{16}$ from which we obtain the spectral position of the resonant modes $\left(\lambda_{0}\right)$, the doublet mode splitting $(\Delta \lambda)$, and the intrinsic cavity $Q$ factor $\left(Q_{i}\right)$. Figure $3(\mathrm{~b})$ shows smooth, continuous tuning is achieved, with a resonance wavelength shift of $\Delta \lambda_{0}=3.8 \mathrm{~nm}$ occurring after $1060 \mathrm{~s}$ of $\mathrm{N}_{2}$ dosing. During the heating phase the $\mathrm{N}_{2}$ shut-off valve is closed, and the wavelength dramatically drops at $T=28 \mathrm{~K}$ as $\mathrm{N}_{2}$ begins to rapidly desorb from the disk surface. ${ }^{17}$ The temperature is further raised to $40 \mathrm{~K}$ to ensure complete $\mathrm{N}_{2}$ removal. Finally, the sample is cooled back down to the starting temperature, at which point $\lambda_{0}, \Delta \lambda$, and $Q_{i}$ have returned to their original values.

The tuning cycle of Fig. 3 was found to be repeatable from run to run, and could be interrupted during the $\mathrm{N}_{2}$ dosing phase to position the cavity mode resonance wavelength with an accuracy of better than $\pm 10 \mathrm{pm}$. Once positioned, for temperatures below $T=20 \mathrm{~K}$ (where $\mathrm{N}_{2}$ desorption is negligible over a time scale of hours ${ }^{17}$ ) we found the cavity mode wavelength to be highly stable. One nonideal side effect of the $\mathrm{N}_{2}$ tuning, evident in Fig. 3(d), is the degradation in the $Q$ factor with increasing $\mathrm{N}_{2}$ adsorption ( $Q$-degradation factors of 2-3 for $4 \mathrm{~nm}$ of tuning were typical for modes of $Q$ $\sim 10^{5}$ ). Several features in the data of Fig. 3 indicate that the optical loss is due to subwavelength optical scattering from the adsorbed $\mathrm{N}_{2}$ film. The clearest indicator is the rapid rise in doublet splitting with wavelength tuning [Fig. 3(c)], a result of increased surface scattering. ${ }^{18}$ Visual inspection of microdisks after large tuning excursions also showed clouding of the top surface. Both observations point to an incomplete wetting of $\mathrm{N}_{2}$ on $\mathrm{AlGaAs}$, and the growth of a rough bulk overlayer consisting of $\mathrm{N}_{2}$ crystallites. ${ }^{19}$

Figure 4(a) shows the wavelength shift versus elapsed time, under varying flow conditions, and for cavity modes of two different microdisks $(D=4.5$, and $2.0 \mu \mathrm{m})$. A simple perturbative analysis relates the cavity mode tuning to the mode's overlap with the disk surface through the equation $\Delta \lambda_{0} \sim \lambda_{0}\left(n_{f}^{2}-1\right) \Gamma^{\prime} t_{f} / 2$, where $t_{f}$ is the film thickness, $n_{f}$ is the film refractive index $\left(=1.21\right.$ (Ref. 20)), and $\Gamma^{\prime}$ is the (linear) modal energy density at the air-disk interface. Such an analysis yields an $\mathrm{N}_{2}$ film thickness of $t_{f} \geq 50 \mathrm{~nm}$ for the measured mode tunings of 2-4 nm. For films of this thickness the perturbative analysis breaks down and one must resort to more exact numerical methods. Finite-element method (FEM) simulations of the mode tuning versus $\mathrm{N}_{2}$ film thickness were performed for the $\mathrm{TE}_{1,20}$ mode of the Downloaded 29 Jan 2007 to 131.215.225.173. Redistribution subject to AIP license or copyright, see http://apl.aip.org/apl/copyright.jsp
$D=4.5 \mu \mathrm{m}$ microdisk, and are plotted in Fig. 4(b) for constant-height $\mathrm{N}_{2}$ coverage of the top and side of the disk (assuming line-of-sight deposition). The measured data are also plotted in Fig. 4(b) versus consumed $\mathrm{N}_{2}$ (estimated from the initial and final chamber pressures, and assuming an exponential decrease in the pressure with time). The simulated $\mathrm{N}_{2}$ thickness is related to the measured $\mathrm{N}_{2}$ consumption with a fixed scaling factor (the sticking coefficient of rare gases to their solids is known to be near unity, ${ }^{21}$ i.e., constant), determined by a least-squares fit to the measured data. From these plots the measured tuning is seen to be independent of flow rate for each of the cavity modes, and the saturation in the tuning rate with increasing layer thickness is well captured by the FEM simulation.

In summary, we have demonstrated that optical fiber tapers can provide an efficient interface for transferring light to and from standard laboratory fiber optics into a micronscale cavity housed in a high-vacuum, cryogenic environment. In addition, we have shown that rare gas adsorption can be used to produce high resolution, continuous tuning of microdisk WGM wavelengths. These two tools are of significant utility to future cQED experiments involving interactions of single QDs with fiber-coupled microdisk cavities. ${ }^{22}$

The authors thank A. Stintz and S. Krishna of the University of New Mexico for materials growth and R. Heron of the Janis Research Company for assistance in the cryostat design.

\footnotetext{
${ }^{1}$ Single Quantum Dots, edited by P. Michler (Springer, Berlin, 2003).

${ }^{2}$ H. J. Kimble, Proc. Stapp Car Crash Conf. T76, 127 (1998).

${ }^{3}$ J. P. Reithmaier, G. Sek, A. Loffer, C. Hoffman, S. Kuhn, S. Reitzenstein, L. V. Keldysh, V. D. Kulakovskii, T. L. Reinecke, and A. Forchel, Nature (London) 432, 197 (2004).

${ }^{4}$ T. Yoshie, A. Scherer, J. Hendrickson, G. Khitrova, H. Gibbs, G. Rupper, C. Ell, Q. Schenkin, and D. Deppe, Nature (London) 432, 200 (2004).

${ }^{5}$ E. Peter, P. Senellart, D. Martrou, A. Lemaître, J. Hours, J. M. Gérard, and J. Bloch, Phys. Rev. Lett. 95, 067401 (2005).

${ }^{6}$ K. Srinivasan, M. Borselli, T. J. Johnson, P. E. Barclay, O. Painter, A. Stintz, and S. Krishna, Appl. Phys. Lett. 86, 151106 (2005).

${ }^{7}$ R. Herrmann, T. Sunner, T. Hein, A. Loffler, M. Kamp, and A. Forchel, Opt. Lett. 31, 1229 (2006).
}

${ }^{8}$ J. C. Knight, G. Cheung, F. Jacques, and T. A. Birks, Opt. Lett. 22, 1129 (1997).

${ }^{9}$ M. Cai, O. Painter, and K. J. Vahala, Phys. Rev. Lett. 85, 74 (2000).

${ }^{10}$ K. Srinivasan, P. E. Barclay, M. Borselli, and O. Painter, Phys. Rev. B 70, 081306(R) (2004).

${ }^{11}$ S. Mosor, J. Hendrickson, B. C. Richards, J. Sweet, G. Khitrova, H. Gibbs, T. Yoshie, A. Scherer, O. B. Shchekin, and D. G. Deppe, Appl. Phys. Lett. 87, 141105 (2005).

${ }^{12}$ S. Strauf, M. T. Rakher, I. Carmeli, K. Hennessy, C. Meier, A. Badolato, M. J. A. DeDood, P. M. Petroff, E. L. Hu, E. G. Gwinn, and D. Bouwmeester, Appl. Phys. Lett. 88, 043116 (2006).

${ }^{13}$ E. R. Abraham and E. A. Cornell, Appl. Opt. 37, 1762 (1998).

${ }^{14}$ K. Srinivasan, A. Stintz, S. Krishna, and O. Painter, Phys. Rev. B 72, 205318 (2005).

${ }^{15}$ From finite-element-method simulations, this mode is identified as a TE (electric field components predominantly in plane), $p=1$, and $m=20$ WGM, where $p$ and $m$ denote the radial and azimuthal mode number, respectively.

${ }^{16} \mathrm{M}$. Borselli, T. J. Johnson, and O. Painter, Opt. Express 13, 1515 (2005).

${ }^{17}$ H. Schlichting and D. Menzel, Rev. Sci. Instrum. 64, 2013 (1993).

${ }^{18}$ M. Borselli, T. J. Johnson, and O. Painter, Appl. Phys. Lett. 88, 131114 (2006).

${ }^{19}$ U. G. Volkmann and K. Knorr, Phys. Rev. Lett. 66, 473 (1991).

${ }^{20}$ S. Pilla, J. A. Hamida, K. A. Muttalib, and N. S. Sullivan, Phys. Lett. A 256, 75 (1999).

${ }^{21}$ H. Schlichting, D. Menzel, T. Brunner, and W. Brenig, J. Chem. Phys. 97, 4453 (1992).

${ }^{22}$ K. Srinivasan and O. Painter, e-print quant-ph/0606142. (2- 